
\title{
25 Research Square \\ Views of medical residents on a research training program: a qualitative study
}

\section{Pamela Abi Khalil}

American University of Beirut Medical Center

\section{Gladys Honein-AbouHaidar}

American University of Beirut Medical Center

\section{Dina El Achi}

American University of Beirut Medical Center

\section{Lara Al-Hakim}

American University of Beirut Medical Center

\section{Hani Tamim}

American University of Beirut Medical Center

Elie A. Akl ( $\nabla$ ea32@aub.edu.lb )

American University of Beirut Medical Center

\section{Research article}

Keywords: Medical residents, research training, mentorship, capacity building, graduate medical education

Posted Date: September 28th, 2020

DOI: https://doi.org/10.21203/rs.3.rs-71467/v1

License: (c) (i) This work is licensed under a Creative Commons Attribution 4.0 International License. Read Full License

Version of Record: A version of this preprint was published at PLOS ONE on January $21 \mathrm{st}, 2022$. See the published version at https://doi.org/10.1371/journal.pone.0261583. 


\section{Abstract \\ Background}

The American University of Beirut Medical Center (AUBMC) developed the Fellowship and Residency Research Program (FRRP) to provide residents and clinical fellows with a supervised and structured research experience.

\section{Objective}

To explore the views of FRRP participants about the training program and how to enhance it.

\section{Methods}

We conducted a qualitative study targeting residents in one of the post-graduate training programs at AUBMC. We invited potential participants through email and via snowball sampling. We continued the data collection until we reached data saturation. We used a thematic analysis to identify emerging themes.

\section{Results}

Four themes emerged from the content analysis: expectations of residents, coordination, mentorship, and capacity building. Residents expected the FRRP to provide them with capacity building activities in conducting research since they perceived themselves as novice researchers. In terms of coordination, few residents complained that the deadlines to submit the deliverables are not evenly distributed across the years, so they suggested moving the deadlines earlier to give more time to address any challenges. In terms of mentorship, participants appreciated the flexibility in choosing the mentor and the ability to choose their own research question in agreement with their mentor, however, they thought that there were few faculty members who conducted research and some lacked commitment due to either being busy or travelling, resulting in the slowing of their progress. In terms of capacity building activities, the participants found the lecture series, both real time and virtual, to be helpful and they were satisfied with the topics of the lectures. Finally, participants pointed out that the FRRP program is very supportive and most of them thought it should be mandatory.

\section{Conclusion}

In order to provide residents and clinical fellows with a supervised and structured research experience, we have built on our findings to introduce several changes to our program such as ensuring the availability and commitment of faculty mentors, providing capacity building activities to the program participants 
and revising the program educational offerings to better cover the ABCs of research using mostly interactive and hands on sessions.

\section{Background}

Early exposure to research experience in undergraduate college, medical students and residents provides an important opportunity to foster one's academic medical career [1] and enhance research capabilities [2]. A survey of psychiatry faculty and residents in the US, reported that $98 \%$ of respondents indicated that research training should be offered to residents [3]. Research curricula instill positive attitudes toward research, particularly among residents, and thus help in achieving the use of evidence at the point of care and increased participation in international research networks [4]. In addition, scholarly productivity based on journal publication is associated with clinical performance during residency training. [5] This suggests that residents who invest substantial efforts in research are not compromised in their abilities to learn medicine and care for patients [5]. Therefore, it is essential to introduce research practices in the early academic career of medical trainees.

In 2011, the faculty of medicine at the American University of Beirut launched the Residency Research Program which is now known as the Fellowship and Residency Research Program (FRRP) [2]. The objective of the FRRP is to provide post graduate trainees with a supervised and structured research experience. The FRRP connects trainees with advisors with whom they work on conceiving, planning, and conducting a research project, and ideally publishing the findings in a peer-reviewed journal [2]. The program is mandatory and targets both categorical residents and clinical fellows. Participants are expected to carry out a research project throughout their residency or fellowship period, and ideally publish it.

Table 1 briefly describes the FRRP (its portal and roles of contributors), while Fig. 1 describes the timeline of participation in the FRRP, using an example of a 3-year residency. Table 2 provides a brief description of the FRRP capacity building activities that cover the 'ABCs of research'. Please note that these details reflect enhancements introduced as a result of this study. 
FRRP portal: An online platform where:

- Residents submit deliverables (e.g., Interest questionnaire, letter of intent, full proposal) and other program requirements (e.g., proof of required training in ethical conduct of research);

- Faculty members review submitted deliverables and provide feedback;

- Coordinator tracks the progress of the residents.

FRRP coordinator:

- Manages the FRRP portal;

- Orients residents to the program, its timeline and its deliverables;

- Follows-up with residents to ensure the timely completion of the research project (through frequent communications and in person meetings);

- Ensures residents identify their FRRP advisor/mentor;

- Ensures the advisor is aware of the progress of the resident's project;

- Organizes the clinical research open house where all graduating residents present their research work in posters and oral presentations.

Advisor:

- Meets with the resident on a regular basis to provide guidance, including on the appropriateness of the research topic;

- Reviews and approves deliverables prior to their submission on the FRRP portal. Makes sure the resident submits the deliverables on time;

- Reviews IRB application and secures its approval;

- Facilitates data acquisition (if applicable);

- Ensures successful completion of the project.

Department representative:

- Makes sure each resident identifies a faculty member as advisor and is working on a feasible scientific research project.

Department coordinator:

- Makes sure each resident identifies a faculty member as advisor;

- Follows up closely with residents to make sure they submit the deliverables before the deadlines. 
Table 2

Brief description of the FRRP capacity building activities that cover the ABCs of research.

- Real time lectures: lectures by faculty members who are experts in the subject matter;

- Recorded lectures: recorded lectures by faculty members accessible on an online platform;

- Biostatistics clinic: Hands-on data analysis/statistical sessions where data analysts provide support, answer questions, and offer advice on issues relating to the statistical analyses of the FRRP projects;

- Opportunity for oral and poster presentations during an "Open house for clinical research" event that mimics national and international conferences.

\section{Appendices}

Appendix 1: interview guide

The start:

Each resident introduces himself, states his area of interest and previous research experience

A- What are your thoughts about the FRRP experience?

Probing:

Do you think that the FRRP provided you with an adequate research experience?

How do you perceive your skills and knowledge in the research steps (literature review, defining a research question, writing a proposal, collecting data, analyzing data, writing a report, delivering oral $\mathrm{PPT}$, preparing a poster) pre-and post-FRRP?

How do you define a mentor?

Do you think your mentor had enough time to allocate for you and to guide you through the process?

Do you think that the FRRP gave you the opportunity to develop a long-term professional relationship with your advisor?

B- How do you perceive a perfect FRRP program?

Probing:

Do you think residents should be given an allocated time to perform research?

Did you take the course on the research process posted on moodle for FRRP residents?

Do you think additional sessions are needed?

Do you prefer hands-on or recoded sessions?

In your opinion, in which steps of the research process residents need help? How can we offer help?

What specific aspects of the program could be improved?

In your opinion, how can we increase FRRP publications? 
The program leadership recently conducted a survey of 103 residents enrolled in the FRRP to evaluate the program.[2] About a fifth (19\%) of participants reported improvement in research-related tasks before and after completion of the program $(P<0.0001)$. Substantive percentages of participants reported either having published $(34 \%)$ or being in the process of publishing their projects $(55 \%)$. At the same time, several participants informally reported some challenges or made suggestions to improve the program. For example, $37 \%(S D=18)$ of participants reported challenges in receiving appropriate supervision.

The objective of this study was to qualitatively explore the views of FRRP participants about the training program and how to enhance it.

\section{Methods}

\section{Overall design and research team}

The study design was that of a focus group discussion. The two team members who conducted data collection (PA and LA) were trained in interviewing and data analysis techniques. Two senior team members (GHA and EAA) with significant experience in conducting and publishing qualitative studies provided oversight and peer observation during the conduct of the interviews. Following the first few interviews, debriefing sessions were held to reflect on the process of data collection and measures for improvement.

\section{Participants}

Our target population consisted of residents in one of the post-graduate training programs at AUBMC. They could be in any year of training except for the 1st year, to ensure they have had enough exposure to the program. We identified potential participants from the FRRP database and invited them by email to participate in the study. In addition, we used the snowball sampling, where we asked the enrolled participants to invite their peers to participate. We recruited participants until we reached thematic saturation in our analysis (see below). The Institutional Review Board (IRB) at the American University of Beirut has approved this study.

\section{Data collection}

We conducted the focus groups during the first half of 2018 in private locations at AUBMC convenient to participants. All participants provided verbal consent and the ethics committee approved this procedure. Discussions were in English, lasted for 50-70 minutes, and were audiotaped. The focus group meetings started with a short description of the study and introductions. Then, the moderators asked open-ended questions with probing questions, using an interview guide (Appendix 1). The discussions explored the following topics: responsibilities and learning experiences of residents, their views, attitudes, challenges, and suggestions. However, we allowed the participants to address other topics, hence allowing new ideas to emerge. We continued data collection until we reached data saturation (i.e., no more new information emerged). 


\section{Data analysis}

Immediately following each discussion, we transcribed the audiotaped recordings and anonymized them. We conducted a thematic analysis where two team members (DA, PAK) worked independently on annotating the transcripts line by line while maintaining a log of emerging themes and exemplary quotes (open coding).

The two team members met with a third investigator (GHA) to share, compare their independent data analysis and resolve any disagreements. Then, they attempted to identify specific themes that summarize the views, attitudes, challenges, and suggestions of the participants. The research team discussed the emerging themes and finalized them. When reporting findings, we supported the narrative by quotes from the participants, and referred to the participants using codes such as P01.

\section{Results}

Participants

We conducted 3 focus groups with a total of 21 residents from different departments at AUBMC, including internal medicine, pediatrics, family medicine, emergency medicine, anesthesiology, and obstetrics and gynecology. Participants were within 2 years of completing their post graduating training.

The content analysis generated four emerging themes (Fig. 2): one related to the expectations of residents, while the remaining three themes related to how the program is working to meet those expectations, specifically: coordination, mentorship, and capacity building. For these three latter themes, we discussed the strengths and challenges related to each.

Expectations of residents

Residents perceived themselves as novice researchers and required capacity building in conducting research. Although 'some had more experience than others'(P01), the consensus was that they lacked the 'ABCs of research'(P02).

Some participants had difficulties identifying important and interesting research questions, and thus were expecting that the program will help them do so.

"I had my own idea and I worked on the literature review from scratch. When I proposed the topic to my attending, he told me this wouldn't be a high impact study" (P03).

Other participants were expecting support for more basic skills.

"We were doing the data sheet and he asked me to do the table and put the values for the research, I wasn't sure, should I put them in a horizontal or vertical way, divide them by sex or just put them all together, I really did not know" (P01)

Program coordination 
Participants pointed to several strengths related to the coordination of the program: requirement to identify a faculty mentor, timeline for completing the research, deadlines to submit deliverables, the close follow-up by the program coordinator, and the use of the portal.

While the program requires the resident to identify a faculty member as a mentor, "as a navigator" (P04), it encourages him/her to approach faculty members with similar research interest.

"I checked my specialty, and the people who are active in this research and I sent emails." (P05)

The timeline to complete the program, and depending on the type of residency, varied between three and five years. Participants highlighted the value of such an extended timeline in allowing enough time for residents to bring their research idea to maturity.

"Some programs might be three, four or five years but overall enough to choose and initiate a study and to change, trial and error, if you don't like your study you can change because you have a lot of time" (P06).

Additional strengths included the deadlines set for submitting the different deliverables and the close follow-up by the FRRP coordinator to complete their research on time.

"Time limits that you have either for 6 or 12 months for your abstract or your proposal in one year should be done. There's a timeline that you must follow. I think that's very good for us specially that doctors get overwhelmed with the time. Follow up is also one of the main advantages that we have" (P07)

Also, having the "portal to upload our proposal... it's a good way" (P04) to communicate back and forth with the program.

In terms of challenges, the participants highlighted their inability to stick to the timeline across the years and the lack of commitment of the mentor toward the research project.

Some participants complained that the deadlines to submit the sequential deliverables are not evenly distributed across the years. For example, the participants pointed that the bulk of the research work as mandated by the deadlines is to be completed during the last year of residency.

"We don't need 2 years to present the proposal, it's a bit too much. We were told about the timeline when we started residency, but we just lose track." (P08)

"It was very long in the beginning and then at the last year the deadlines become very intense.

Condensed... And then we got stuck during the last year" (P09)

As a result, if residents faced unexpected delays as they are conducting research, then they run into the risk of not being able to graduate. 
"If something went wrong during data collection, in the third year, I have to go back from the beginning, so how am I going to make any progress in a new project if I'm going to start in mid third year and I'm not allowed to graduate if I don't have a research project" (P01)

As recommendations, and to address the timeline challenge, participants suggested moving the deadlines earlier to give more time to address the challenges, if any, and still be able to graduate on time. Another suggestion was to have two residents work on the same research project instead of only one.

"Make the deadlines sooner, for example for the proposal, it was 2 years to get the proposal, it's a bit too much... (P09)

"Shift everything 6 months"(P04)

"Shift everything back. Make the deadlines closer" (P09)

"2 person/project structure: less burden than that experienced by one person. You would decrease the workload, and at the same time you would have your name in publication" (P01)

Participants also suggested having more coordination at the departmental level. Some suggested that the FRRP coordinators monitor progress and check on the challenges of the residents. Others suggested having in every department, a clinician scientist well versed in research as "a reference person" (P04), to coordinate and monitor the progress of residents.

"In every unit we have a chairman so why don't we have a person who is the head of research in the department and asks people where you are now" (P08)

"And you would rather that person be an attending?"(P10)

"Yes because, "maha" only follows the residents. maybe this champion meets with the residents and attending at the same time and ask them where are you at." (P08)

"A closer follow up. Where are we right now what did we do so far, a close face to face interaction, a regular follow up" (P02)

Mentorship

Participants appreciated the flexibility in choosing the mentor.

"Most of them didn't reply. I went to the one who replied and talked to him, he was very cooperative"(P05)

They typically based their choice of mentor on the experience of their seniors with the different attendings.

"I asked my seniors whom they worked with and who was most dedicated and engaged in research and who is most cooperative to work with and who finds time most to work" (P11) 
Senior residents also encouraged their junior colleagues to seek mentors who have their own research assistant.

"If someone asked me what you think about choosing this mentor over someone else, if he has a research assistant I will say go for it." (P04)

Participants highlighted, as one of the strengths of the program, the ability to choose their own research question in agreement with their mentor.

"Again as P07 said we are always more than encouraged to choose our own topic rather than going to the mentor and asking them what they have as a potential project" (P04)

In terms of challenges, participants thought there were not enough faculty members who conducted research.

"FRRP program tells you that you can choose whoever you want but when you get to actually doing it you are limited by the specialty and sometimes one person within that specialty" (P12)

A second challenge was the lack of commitment of some mentors due to either being busy or travelling, hindering the progress of their research project.

"Sometimes we really try to be on track, we always send them that we need to finish but due to his busy schedule or when he's abroad for a conference, he keeps on postponing the issue. It is important to stress that there are deadlines to be part of the whole procedure as well" (P02).

Some residents complained that their mentors were very slow in responding to their requests.

"Took more than 6 to 7 months to get it back."(P09)

Also, some mentors can be overly busy with other mentees.

"Mentors are not available or already saturated with their mentees, so we find ourselves limited." (P03)

Some participants felt intimidated by their mentors and indicated "worrying about disappointing" them (P04). This resulted in "acting like you know how you are doing" (P13), and residents spending time figuring out things on their own.

"Sometimes I would go on the internet, on google and YouTube" (P03)

"An attending asks have you done it before? if you say no, he won't give it to you. If you've read it maybe and seen videos a million times but you show confidence in front of him, he would trust you" (P13)

Most feel embarrassed to ask "silly questions" (P04) to their mentors to avoid being judged as not competent enough for the program so they "rather not ask" (P01). 
"My mentor asked me where you are now with the research, I said it's in progress and that I'm trying to get the IRB and that was it. He writes FRRP in progress, and honestly at some point, you might feel shy to ask the attending about something." (P01)

While residents had the flexibility to choose their own research questions, they may end up working on a question proposed by the mentor. However, this is not problematic for the residents given the "shared interest" they have with the attending they chose (P04). In addition, the mentors have more research experience and can suggest a research question that is likely to be important and influential.

"They [mentors] know more than us, they have their experiences and can help us." (P06)

"The idea of having a topic by ourselves shouldn't be the focus for FRRP it's more like to learn how to start a research from scratch. Regardless of the topic by itself" (P07)

In terms of recommendations, more than one participant suggested that FRRP develops for each department a list of attendings that are active in research and are good mentors in order to guide residents in their choices;

"The list of advisors, do not include all people who are dedicated for research, because we know in every specialty the attending who is very busy doing always research" (P07)

"You are asking FRRP to indicate who are the good attendings." (P12)

Also, FRRP should be looking at the mentorship workload. If mentors have too many residents that are working with them, then they need to direct residents to others

"Ensure that the mentor is available to the mentee and you're proposing one way is to stick the number of mentees to that person" (P12)

Capacity building

Most residents were concerned about meeting the timeline set by their mentors mainly due to their illpreparedness in conducting research. However, when considering the specific capacity building activities, the participants found the lecture series, both real time and virtual, to be helpful. In addition, they were satisfied with the topics of the lectures.

"The topics chosen are very good; manuscript writing, data analysis, SPSS, proposal, and I think they are very good. Instead of increasing the series of lectures, maybe that time will be allocated for research. I don't think adding on the very good topics will add any benefit or time efficiency to the residents." (P06)

They asked for small group discussions where residents will be given the opportunity to share their experiences, challenges and learn from each other measures on how to overcome them.

"Certain things may actually rise; several people would actually agree and it also happened with me" (P02) 
"When it's a small group it's easier to understand and we can teach other it would be fine." (P08)

They also appreciated posting the lectures on the web.

"We have sessions on moodle, that you can access. I think they are useful as a reminder after attending the lecture and we're having any question we can use it." (P14)

The participants perceived the "clinical research open house" event as an opportunity to showcase their work and get prepared for presenting at national or international conferences.

"It's good to be exposed to this kind of work because many residents are speakers abroad, they prepare posters, and go to national conferences and meetings so it's like an exercise." (P11)

"The open house actually mimics the conferences"(P08)

"yeah" (P11)

"It's a good opportunity for you then to be part of it and experience that whole poster presentation and oral presentation" (P08)

"Exactly"(P11)

Finally, participants pointed out that the FRRP program is very supportive and resourceful.

"We set meetings whenever we have issues. They always find time available. Whenever the attending is there, we can ask questions, we just refer to them, what should I do about this, and they directly give us an answer. Last time I had a question for [mentor], she picked up the phone and called [FRRP person]. Another time someone else and she solved the issue, it was very easy." (P11)

In terms of challenges, some participants thought that the capacity building sessions were not interactive enough.

But it's not interactive

Also, some participants reported not taking advantage of the recorded sessions because they either forget or do not have the time.

“But honestly we don't check them"(P04)

Other participants had time management issues.

"The only time we have dedicated to education is our morning lecture, from 7 till 7:30 am this is the only time, I'm sure we can make it at another time otherwise, I don't know" (P11)

In terms of recommendations, there was a suggestion to add an initial session to introduce research and to make the sessions mandatory for every first-year cohort. 
"I think there has to be something at the beginning to introduce research" (P01)

Further, participants suggested having "a standard like first 6 months to a year where all the specialties are to attend 2 or 3 workshops" (P04). The topics should cover all components of research.

When we asked the participants whether FRRP should be mandatory or optional, most participants thought it should be mandatory.

"even if you are not going to do research later on, the FRRP helps you understand how research is conducted and how it should be interpreted" (P04)

There was further discussion on making certain components of the program mandatory.

"We have to generalize the $A B C$, like we said grand rounds for everyone, and then after a certain point this has to become personalized, individualized, so that there is this one reference person that whenever you have any question you can just go and they are ready to help" (P13)

Two participants indicated that because FRRP is mandatory, they enrolled in the program.

"I think that FRRP is not something that would help me it's more of something forcing me to do a research before I graduate... it's just something I have to do because it is mandatory to do it" (P13)

While others, thought they would rather have it "optional" (P01).

\section{Discussion}

\section{Summary}

The objective of this study was to explore the views of FRRP participants about the training program and how to enhance it. In terms of expectations, the residents perceived themselves as novice researchers and required support with basic skills. The most common reported difficulty was the identification of important and interesting research questions. In terms of the program coordination, participants appreciated having a timeline and deadlines, the expectation of a close follow-up by the mentor, the close follow-up by the program coordinator, and the use of the portal. However, some complained about the tight timeline. In terms of mentorship, participants appreciated the flexibility in choosing the mentor and their own research question. However, they complained about having few faculty members who conducted research and about the lack of commitment of some mentors. In terms of capacity building, the feedback was overall positive, and the participants pointed out that the FRRP program is very supportive and resourceful. Participants also reported some challenges (e.g., the capacity building sessions were not interactive enough). Participants made practical recommendations to address these different challenges.

Strengths and limitations of the study 
The main strength of this study is the use of several approaches to increase the rigor of our study, including training of interviewers, peer observation, debriefing, and duplicate data analysis. Also, this is the first study we are aware of to assess residents' views about training in medical research.

One limitation of the study is that we were not successful in recruiting participants from all the departments at AUBMC. However, we suspect that the views of those who participated are representative of our target population.

\section{Comparison to similar studies}

We were not able to identify qualitative assessments of programs like ours. However, we did identify studies describing similar programs [6] [7]. Kanna et al. assessed a dedicated "research rotation" at a university hospital in New York city to teach residents how to design and implement a successful research project [6]. They reported increased participation of residents in scholarly activities and their perception of research as being essential for practice of medicine. In another study, Hurst et al. described the research training of pediatric residents and clinical fellows as part of the Duke Pediatric Research Scholars Program for Physician-Scientist Development (DPRS) [7]. Participants were able to secure internal (department and university-wide) and external funding and publish articles in peer-reviewed journals. Dagher et al. reported on a similar program conducted at the American University of Beirut in Lebanon but amongst undergraduate students [1]. Under this program, students volunteer to work with faculty members on clinical and basic science research, to learn about research firsthand. The authors reported that participants have co-authored publications in peer-reviewed journals with their respective advisors and rated the program positively.

\section{Implications for practice}

Our findings emphasize the importance of understanding and trying to meet the expectations of residents when establishing research training programs. Indeed, we have built on these findings to introduce several changes to our program. Example of changes include modifying the timeline and deadlines of the program (Fig. 1) and enhancing the program portal. A major implication of our findings is ensuring availability and commitment of faculty mentors and when needed, offer them training in mentorship skills. As a result, the program has encouraged coordination at the departmental level particularly for ensuring an optimal resident-to-mentor matching. Another implication is importance of providing capacity building activities to the program participants. In our case, we have revised the program educational offerings to better cover the $A B C$ s of research using interactive and hands on sessions. It is important to conceive such programs as a major investment for participants and departments alike, aiming to enhance research productivity [8].

Implications for future research

It would be important to have a follow up study, with both participants and advisors, to assess how the changes introduced as a result of this study have changed the research culture and the research 
productivity during and following training. More generally, research is needed to explore optimal ways to improve the research experience of clinical trainees.

\section{Declarations:}

\section{Declarations}

\section{- Ethics approval and consent to participate}

The Institutional Review Board (IRB) which is part of the Human Research Protection Program (HRPP) at American University of Beirut has approved this study. Protocol Number: IM.HT.08/SBS-2017-0273. Informed verbal consent was obtained from all participants and the institutional ethics committee approved this procedure.

\section{- Consent for publication}

Verbal consent was obtained from all participants involved in the focus group discussions

\section{- Availability of data and materials}

Data cannot be shared publicly because of confidentiality and privacy concerns. The American University of Beirut Institutional Review Board (IRB) approved the study, and the consent document the IRB approved assured participants that their data would not be shared beyond the research team and as aggregated in publications. The deidentified data for the study may be made available to investigators who contact AUB in accordance with institutional policies. Please note that AUB policies require AUB investigators to retain custody of research data, unless Non-Disclosure Agreements (NDA) have been signed prospectively with investigators/collaborators in other institutions. You can also contact AUB IRB office (irb@aub.edu.lb) for any additional inquiries related to human subjects' data for research purposes.

\section{- Competing interests}

- Elie Akl directs the Clinical Research Institute, under which the program that was evaluated operates.

- Hani Tamim directs the Fellowship and Residency Research Program, the program that was evaluated.

- Abi Khalil coordinates the Fellowship and Residency Research Program, the program that was evaluated.

- All remaining authors have nothing to disclose.

\section{- Funding}

Not applicable 
EAA and HT conceived the study. EAA and LAH designed the study. PAK coordinated the study. EAA had full access to all the data in the study and takes responsibility for the integrity of the data and the accuracy of the data analysis. PAK and LAH collected the data. GHA and EAA provided oversight and peer observation during the conduct of the interviews. DA anonymized and transcribed the recordings. DA and PAK annotated the transcripts. GHA identified the specific themes. The research team discussed the emerging themes and finalized them. PAK and EAA analyzed and interpreted the data. PAK wrote the first draft of the manuscript with EAA. GHA wrote the first draft of the results. All authors critically revised the manuscript and approved the final manuscript. EAA affirms that this manuscript is an honest, accurate and transparent account of the study being reported; that no important aspects of the study have been omitted; and that any discrepancies from the study as planned have been explained.

\section{- Acknowledgments}

We acknowledge the contribution of the volunteer Dina El Achi under the Medical Research Volunteer Program (MRVP) at the American University of Beirut and Zeina Akiki and Rola El Rassi for their contribution to proposal writing.

\section{References}

1. Dagher, M.M., et al., Medical Research Volunteer Program (MRVP): innovative program promoting undergraduate research in the medical field. BMC Med Educ, 2016. 16: p. 160.

2. Tamim, $\mathrm{H}$. , et al., Structure and evaluation of a residency research program in a university hospital. BMC medical education, 2019. 19(1): p. 406.

3. Fitz-Gerald, M.J., et al., Psychiatry Residents' Participation in Research. Academic Psychiatry, 2001. 25(1): p. 42-47.

4. Smith, M., Research in residency: do research curricula impact post-residency practice? Fam Med, 2005. 37(5): p. 322-7.

5. Seaburg, L.A., et al., Associations between resident physicians' publications and clinical performance during residency training. BMC Medical Education, 2016. 16(1): p. 22.

6. Kanna, B., et al., The research rotation: competency-based structured and novel approach to research training of internal medicine residents. BMC Med Educ, 2006. 6: p. 52.

7. Hurst, J.H., et al., Cultivating Research Skills During Clinical Training to Promote Pediatric-Scientist Development. Pediatrics, 2019. 144(2).

8. AUBMC. AUBMC 2020 vision. [cited February 2020; Available from: http://www.aubmc.org/2020/Pages/vision2020.aspx.

\section{Appendices}

Appendix 1: interview guide 
The start:

Each resident introduces himself, states his area of interest and previous research experience A- What are your thoughts about the FRRP experience?

Probing:

Do you think that the FRRP provided you with an adequate research experience?

How do you perceive your skills and knowledge in the research steps (literature review, defining a research question, writing a proposal, collecting data, analyzing data, writing a report, delivering oral PPT, preparing a poster) pre- and post-FRRP?

How do you define a mentor?

Do you think your mentor had enough time to allocate for you and to guide you through the process?

Do you think that the FRRP gave you the opportunity to develop a long-term professional relationship with your advisor?

B- How do you perceive a perfect FRRP program?

Probing:

Do you think residents should be given an allocated time to perform research?

Did you take the course on the research process posted on moodle for FRRP residents?

Do you think additional sessions are needed?

Do you prefer hands-on or recoded sessions?

In your opinion, in which steps of the research process residents need help? How can we offer help?

What specific aspects of the program could be improved?

In your opinion, how can we increase FRRP publications?

\section{Figures}




\section{First Year}

\begin{tabular}{|l|}
\hline June \\
\hline July \\
\hline August \\
\hline September \\
\hline October \\
\hline November \\
\hline December \\
\hline January \\
\hline February \\
\hline March \\
\hline April \\
\hline
\end{tabular}

Second Year

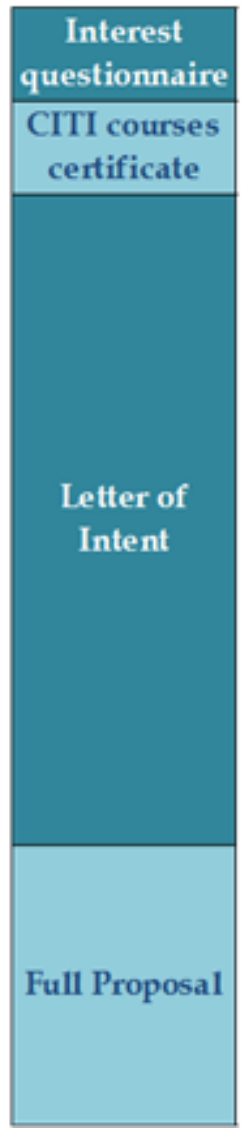

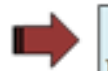

June

I methodology

July

August

September

October

November

December

January

February

March

\begin{tabular}{|l|}
\hline April \\
\hline May \\
\hline
\end{tabular}

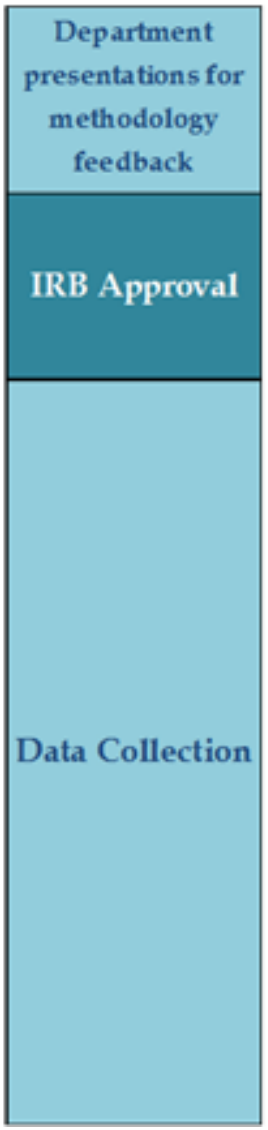

\section{IRB Approval}

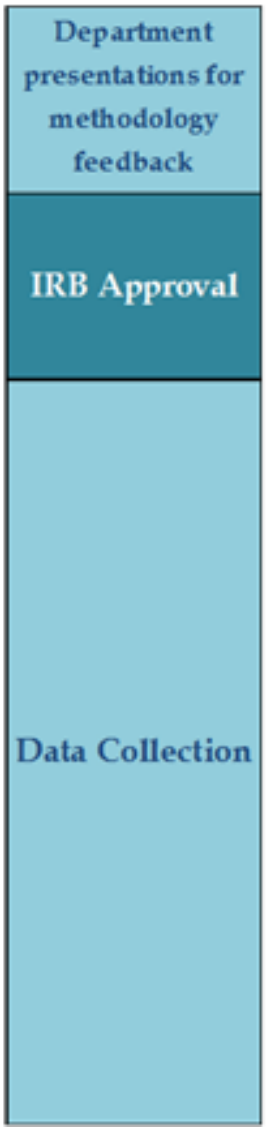

Data Collection
Third Year

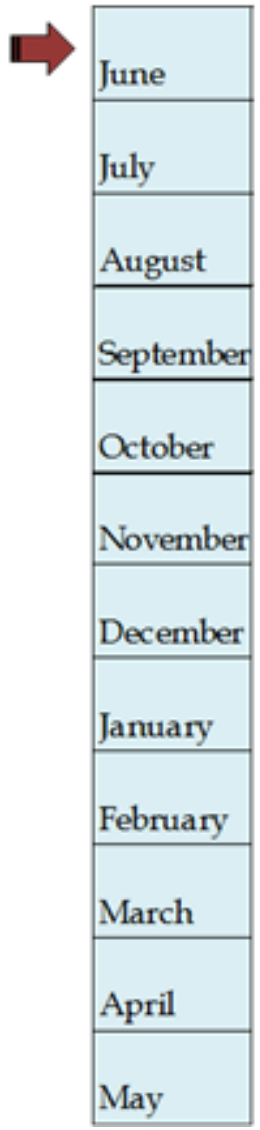

June

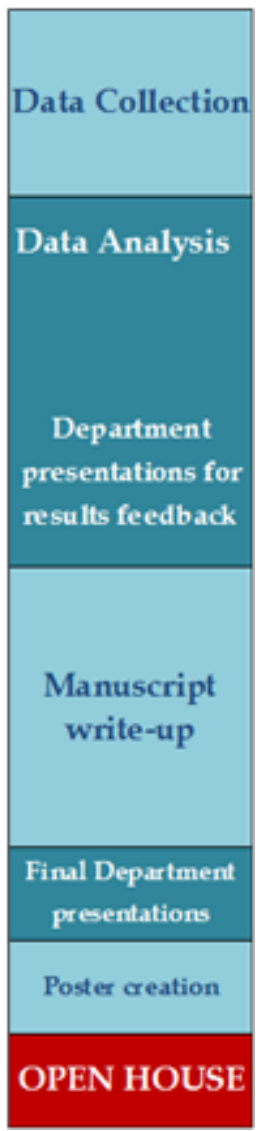

\section{Figure 1}

Timeline for residents (in a 3-year residency) participating in the FRRP to complete their projects.

\section{FRRP}

\section{Coordination}

Strengths/limitations

\section{Mentorship}

Strengths/limitations

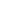


Schematic representation of the four emerging themes

Page 19/19 